\title{
How many children meet dietary targets for both fat and sugar intake? A national survey of dietary intake of children in Scotland
}

\author{
J. I. Macdiarmid ${ }^{1}$, L. C. A. Craig ${ }^{2}$, J. Loe ${ }^{2}$, L. F. Masson ${ }^{2}$ and G. McNeill ${ }^{1,2}$ \\ ${ }^{1}$ Rowett Institute of Nutrition and Health, University of Aberdeen and ${ }^{2}$ Division of Applied Health Sciences, \\ University of Aberdeen, Aberdeen, $U K$
}

Dietary targets are set for monitoring within the general population but they can provide a useful indication of the proportion of individuals meeting the dietary targets, as well as whether individuals are achieving a combination of the targets. The aim of the present study was to estimate the proportion of children in Scotland with intakes meeting the dietary targets of $\leq 35 \%$ food energy from total fat, $\leq 11 \%$ food energy from SFA and $\leq 10 \%$ total energy ( $\leq 11 \%$ food energy) from non-milk extrinsic sugar (NMES) as set out in the Scottish Diet Action Plan ${ }^{(1)}$.

The Survey of Sugar Intake among Children in Scotland ${ }^{(2)}$ conducted in 2006 assessed the dietary intake of a national sample of children across Scotland using a 140-item FFQ. The survey achieved a response rate of $67 \%$, with usable data for $1391 \mathrm{children}$ aged 317 years. Dietary data were used to estimate the percentage of children meeting dietary targets for total fat, SFA and NMES intake.

As a population these children achieved the dietary target for total fat, but approximately one-third of children had intakes $>35 \%$ energy from total fat (see Table). Only $10 \%$ of children met the targets for either SFA or NMES. There was no difference between boys and girls in the percentage meeting these targets. Younger children (3-11years) were more likely than older children (12-17 years) to meet targets for NMES $(12.3 \% v .7 .2 \% ; P=0.02)$ but less likely to meet targets for SFA $(7.5 \% v .14 .3 \% ; P<0.001)$. Of the children $59 \%$ met only one of the dietary targets and $<2 \%$ of children met all three dietary targets. All the children that met the target for SFA also met the target for total fat intake, but only $15 \%$ of those who met the total fat target also met the SFA target. A subsample of 156 children also completed a diet diary, which supported these findings and provided examples of the types of meals eaten across the day that achieved the targets.

\begin{tabular}{lccc}
\hline & $\begin{array}{c}\text { Percentage } \\
\text { Nutrient targets }\end{array}$ & \multicolumn{2}{c}{ Population intake (\% food energy) } \\
\cline { 2 - 4 } Total fat ( $\leq 35 \%$ food energy) & 68.8 & Mean & $95 \%$ CI \\
SFA ( $\leq 11 \%$ food energy) & 10.2 & 13.8 & $32.7,33.2$ \\
NMES ( $\leq 11 \%$ food energy) & 10.3 & 17.4 & $13.7,14.0$ \\
Meeting all three targets & 1.6 & - & $17.0,17.8$ \\
Meeting any two targets & 12.7 & - & - \\
Meeting only one target & 59.0 & - & - \\
Meeting none of the three targets & 26.7 & - & - \\
\hline
\end{tabular}

Only a very small proportion of children met all three dietary targets and it is likely that this proportion would reduce further if other dietary targets were added (e.g. salt, complex carbohydrates). To ensure a healthy balanced diet it is important not to consider the dietary targets in isolation. In terms of the health of a nation future monitoring should take into account the intake of combined nutrients by individuals in addition to assessing the population mean for each nutrient. The fact that all the children that meet the SFA target also met the target for total fat has implications for simplifying healthy eating advice to focus on SFA rather than total fat.

Work funded by the Food Standards Agency, Scotland.

1. The Scottish Office (1996) Eating for health: a diet action plan for Scotland. http://www.scotland.gov.uk/library/documents/diet-00.htm

2. Food Standards Agency (2008) Survey of Sugar Intake among Children in Scotland. www.food.gov.uk/scotland/scotnut/scotsug 